\title{
Procedural sedation and analgesia in children undergoing digestive endoscopic procedures - paediatrician or anaesthesiologist?
}

\author{
Alicja Bartkowska-Śniatkowska ${ }^{1}$, Jowita Rosada-Kurasińska ${ }^{1}$, Iwona Ignyś², Małgorzata Grześkowiak ${ }^{3}$, \\ Marzena Zielińska ${ }^{4}$, Agnieszka Bienert ${ }^{5}$ \\ ${ }^{1}$ Department of Paediatric Anaesthesiology and Intensive Therapy, Poznan University of Medical Sciences, Poznan, Poland \\ 2Department of Paediatric Gastroenterology and Metabolic Diseases, Poznan University of Medical Sciences, Poznan, Poland \\ ${ }^{3}$ Department of Teaching Anaesthesiology and Intensive Therapy, Poznan University of Medical Sciences, Poznan, Poland \\ ${ }^{4}$ Department of Anaesthesiology and Intensive Care, Paediatric Intensive Care Unit, Wroclaw Medical University, Poland \\ ${ }^{5}$ Department of Clinical Pharmacy and Biopharmacy, Poznan University of Medical Sciences, Poznan, Poland
}

Prz Gastroenterol 2014; 9 (2): 77-81 DOI: $10.5114 /$ pg.2014.42501

Key words: conscious sedation, endoscopic procedures, children, paediatrician, anaesthesiologist.

Address for correspondence: Alicja Bartkowska-Śniatkowska MD, PhD, Department of Paediatric Anaesthesiology and Intensive Therapy, Poznan University of Medical Sciences, Karol Jonscher's University Hospital, 27/33 Szpitalna St, 60-572 Poznan, Poland, phone: +48 618491 478, fax: +48 618491 486, e-mail: asniatko@ump.edu.pl

\begin{abstract}
Endoscopic procedures of the gastrointestinal tract were successfully introduced into paediatric practice in the 1970s. Recent expansive development has become useful for improvement of both diagnosis and treatment in many children with gastrointestinal diseases. Most of these procedures are performed under procedural sedation (PSA) knowing anatomical, physiological and psychological differences and requiring good experience from the paediatrician and anaesthesiologist. These principles help to provide the procedure safely and minimise adverse events, which are greater the smaller the child is. Procedural sedation and analgesia in healthy children can be performed by a paediatrician, but children with congenital defects and serious coexisting diseases $(A S A \geq I I)$ and also during the usage of anaesthetics (e.g. propofol), should be managed by an anaesthesiologist.
\end{abstract}

\section{Introduction}

In recent years there has been a significant increase in the importance of sedation applied to children during diagnostic and therapeutic procedures, including endoscopic treatment of the gastrointestinal tract, which is commonly defined as Conscious Sedation or Procedural Sedation \& Analgesia (PSA). The expansive development of invasive therapeutic methods has enforced the improvement or introduction of new methods in children's anaesthesiological practice [1]. Some of those practices found many supporters also among non-anaesthesiologists, but usually among the population of adult patients, who are at lesser risk of complications than children [2]. At present the mortality rate of adult patients related with anaesthesia is very low, i.e. $0.5-0.9$ per 10,000 cases of anaesthesia, and it is even lower in 1-day treatments, i.e. 0.012-0.029 per 10,000
[3]. However, children are a different group in terms of their anatomical and physiological maturity, where the risk of adverse reactions is much greater than in adults. Due to this fact, in this group of patients, not only deep sedation and general anaesthesia but also procedural sedation is carried out by anaesthesiologists, because it minimises the risk of complications, and if they occur, it guarantees appropriate proceedings. The percentage of cardiac arrest in the perioperative period is still high in children, i.e. 1.4 per 10,000 cases of general anaesthesia $(0.014 \%)$. The mortality rate in this group of patients is even higher and every fourth child dies after cardiac arrest (24\%), whereas the other children (15-30\%), whose lives have been saved, suffer from severe irreversible neurological disorders $[4,5]$.

The aim of the study is to present the latest reports on the application of conscious sedation to children during endoscopic treatment of the alimentary tract. 
Another important aspect of this publication is an attempt to answer the question of whether this procedure can be carried out by a paediatrician or if it should be carried out by an anaesthesiologist.

\section{Definitions}

Conscious or procedural sedation (PSA) consists of two methods: minimal sedation and conscious sedation. Minimal sedation consists of pharmacological limitation of the brain activity and its cognitive and coordinative functions. The main goal of this sedation is to suppress anxiety and fear but to retain full capability of responding to verbal stimuli. Therefore, it is also called an anxiolysis. It is not accompanied by respiratory or circulatory disorders. This method is applied to older children, especially in painless diagnostic procedures. Conscious sedation is the state where the patient's consciousness is limited and/or suppressed, but usually there is verbal contact with the patient, who reacts to verbal ortactile stimuli. It is an important aspect to achieve amnesia, especially when procedures need to be repeated. Then it is possible to achieve the child's acceptance. The patient's reflexes are retained and they usually breathe spontaneously, although the younger the child is, the more often there are problems in keeping airways open and maintaining normal ventilation. The function of the circulatory system is usually not disordered. This method is broadly dedicated to infants and small children, children who do not cooperate with the physician, and in painful endoscopic procedures. However, the method is burdened with the risk of passage into deep sedation without noticeable clinical symptoms, which is usually related to the different pharmacokinetic and pharmacodynamic properties of those drugs in infants and children. For this reason, it is necessary for an anaesthesiologist, ideally a paediatric anaesthesiologist, to be present during sedation to carry it out safely and, at the same time, to avoid adverse reactions.

Deep sedation consists of pharmacological suppression of brain activity, which is much deeper than in PSA. It is possible to receive a verbal response only after multiple repetitions of verbal or tactile stimuli. The patient's spontaneous breathing can be impaired and may require assistance in maintaining a patent airway. This type of sedation is recommended for painful and discomforting procedures and in situations when it is necessary to immobilise the patient.

\section{Premedication visit. Risk assessment during the sedation of infants and children}

Patients' state of health, congenital disorders and concomitant diseases are assessed according to the six-category scale of the American Society of Anaesthesiologists (ASA). According to the scale, the higher the category is, the more severe the patient's state of health is and the higher the risk of adverse effects is. The risk of complications during sedationof children is usually related to circulatory disorders (37\%), drug reactions (32\%), respiratory disorders (20\%), faulty/ inappropriate equipment (7\%) and other events (4\%). The higher the child patient's category according to the ASA scale is (ASA $\geq I I I)$, the greater the risk of complications is, although $33 \%$ of the children who had cardiac arrest in the perioperative period had been assessed as category I or II before the surgery [6]. The assessment of the patient before beginning the procedure and the preparation of the child for it are the factors which may influence the number of complications. Insufficient and inappropriate assessment of the child before qualifying for sedation is the cause of numerous hazards. Infants and small children are a group of patients who may experience circulatory collapse and respiratory failure unexpectedly and very rapidly. The younger the child is, the more disorders there are, and they are more severe if they are accompanied by multiple organ disabilities. They occur more often with malformation syndrome and other concomitant diseases. The greater the PK/PD (pharmacokinetic and pharmacodynamic) differences between the applied drugs are, the sooner these disorders occur. Procedural sedation applied to children became initially widespread in emergency departments $[7,8]$. A large number of complications and adverse events was observed in this group of patients (17.8\% of cases) [9]. Most of them were related to respiratory disorders. As many as 1 out of 200 children who were subjected to PSA needed to have airway opened and required support ventilation [10]. In view of these hazards, it is particularly important to initially identify the patients at high risk of occurrence of respiratory complications. This will enable the choice of the optimal procedure both during sedation and endoscopy [11]. Thus, although the ASA scale is recognised around the world, it does not seem to be sufficient due to its incomplete assessment of the airways in paediatrics, especially when disorders become intensified in the sick child's recumbent position or they occur in difficult-to-intubate patients $[12,13]$. Another important aspect is to recognise potential difficulties in keeping airways open during sedation due to anatomical defects (e.g. Pierre Robin syndrome, Marfan syndrome) and systemic diseases (e.g. Down syndrome, mucopolysaccharidosis) [10]. It must be remembered that until the age of five the anatomy of the child's airway is naturally different the adult's. The tongue is big, the larynx is funnel-shaped, the subglottic regionis the narrowest 
section, the epiglottis is long, the trachea and bronchi have high airway resistance, the intercostal muscles are poorly developed and the diaphragm is the main respiratory muscle [14]. The Mallampati classification and direct laryngoscopy are good and useful tests predicting difficulties in maintaining normal airway patency (Table I) [13]. The examination procedure consists of the patient opening their mouth and putting their tongue out for visual examination of the posterior pharyngeal wall or direct assessment of the vocal cords. Younger or non-cooperative children can be examined in the recumbent position with a spatula pressing the tongue [15].

The third significant complication may be laryngospasm, which is common until the age of five, especially if there is a concomitance of upper respiratory tract infections and gastroesophageal reflux. In recent years, European paediatric scientific societies have strongly recommended postponement of an invasive medical procedure for up to 14 days, if the patient suffers from a respiratory tract disease, due to the higher risk of respiratory complications. It does not apply to non-allergic or allergic rhinitis, but it applies to cough, mucopurulent exudate or fever, which increase the risk of laryngospasm (partial closure of the larynx with stridor), which cannot be eliminated with different manual manoeuvres such as jaw-thruster chin-lift manoeuvres. Total closure is more dangerous as it causes the risk of direct life hazard to the child. For endoscopists it may be significant to take into consideration gastroesophageal reflux, the ejection of saliva and secretion of the oral cavity and pharynx, and irritation of the posterior pharyngeal wall, which is related with it and which may lead to laryngospasm [16]. It is necessary to stress the fact that anaesthesiologists are much more skilled than paediatricians in their ability to apply appropriate manual manoeuvres and pharmacological treatment.

\section{Feeding}

In view of the above-mentioned hazards, the problem of feeding children with endoscopic medical procedures seems to be of double significance. On the one hand, the risk of aspiration increases if sedation is too deep, and on the other hand, it increases if there is concomitant gastroesophageal reflux or intestinal obstruction. It is unquestionable that the child needs to fast before the procedure, but on the other hand, fasting too long is unfavourable to the child. Water or tea may be given $2 \mathrm{~h}$ before PSA, breast milk $-4 \mathrm{~h}$ before and solid food (including artificial milk mixtures) $-6 \mathrm{~h}$ before treatment. As far as children at greater risk of aspiration are concerned, the time should be extended to $6 \mathrm{~h}$, regardless of the type of liquids or food.
Table I. Mallampati Scale and Direct Laryngoscopy Scorring System

\begin{tabular}{cc} 
& Mallampati Scale \\
\hline Grade 1 & Whole of glottis visible \\
\hline Grade 2 & Glottis incompletely visible \\
\hline Grade 3 & Epiglottis but not glottis visible \\
\hline Grade 4 & Epiglottis not visible \\
\hline Grade 1 & Full view of the glottis \\
\hline Grade 2 & Partial view of the glottis or arytenoids \\
\hline Grade 3 & Only epiglottis visible \\
\hline Grade 4 & Neither glottis nor epiglottis visible
\end{tabular}

\section{Laboratory investigations}

Around the world there are a great number of opinions about carrying out laboratory investigations on children before they are subjected to endoscopic procedures of the alimentary tract. The range of investigations depends on the extent of the procedure, type of qualification, concomitant diseases and the child's current state of health. Usually, children ranked as ASA I-I may undergo PSA without laboratory investigations. If there is a risk of complications or the child's state has been ranked as ASA III or higher, it is necessary to carry out extra tests, such as complete blood count, serum electrolyte concentrations or coagulation system parameters, or even to identify the blood type. It is good practice to receive an opinion about the patient's current state of health from the appropriate consultant when the child has a chronic disease.

\section{Premedication}

The final element of the qualifying interview before PSA is to determine the indications for pharmacological premedication. This gives a possibility to achieve adequate emotions and to reduce stress or anxiety. Children do not understand most medical procedures, they find them hard to accept, especially if they need to be repeated many times. Effective premedication causes sedation, suppresses anxiety, improves cooperation with the patient, and reduces vegetative reflexes and excessive salivation, which are so typical of infants and toddlers [17]. At present, the latest recommendations suggest the application of oral benzodiazepine, preferably midazolam, $30 \mathrm{~min}$ before the procedure, at a dose matching the patient's age, i.e. a higher dose of $0.75 \mathrm{mg} / \mathrm{kg}$ of body weight for children aged 1-6 years and a lower dose of 
$0.3 \mathrm{mg} / \mathrm{kg}$ of body weight for teenagers. Infants usually need intermediate doses, i.e. $0.5 \mathrm{mg} / \mathrm{kg}$ of body weight. However, from the authors' own experience, oral administration of the drug is not preferable in gastroscopic examinations due to the long presence of the drug in the lumen of the stomach. Therefore, intravenous administration of appropriately corrected doses is preferable. $\alpha 2$ Receptor agonist drugs are more and more often applied, e.g. dexmedetomidine and clonidine, but the former has an 8-times stronger specific effect on $\alpha 2$ receptors than the latter. Both drugs produce the effect of sedation, suppress anxiety, and reduce pain and vegetative reactions. However, the most important fact is that they do not cause concomitant respiratory or circulatory disorders. The oral doses are $2.5 \mu \mathrm{g} / \mathrm{kg}$ for dexmedetomidine and 4-5 $\mu \mathrm{g} / \mathrm{kg}$ for clonidine. Both midazolam and these two drugs can be successfully administered nasally, which is significant in endoscopic procedures of the upper alimentary tract. However, the vegetative activity is lower and analgesia is stronger in the children who received $\alpha 2$ receptor agonist drugs [18]. In most cases, parents' presence would be sufficient, but it is not always possible due to the administrative conditions and both parents' and their children's psychological predispositions. However, there are enthusiasts of some very modern methods, e.g. introducing clowns into paediatric departments, which results in much better sedation and reduction of the drug dose administered to small children than after the application of different drugs [19].

\section{Procedural sedation and analgesia drugs}

Midazolam seems to be the best drug in PSA carried out by non-anaesthesiologists. It is a benzodiazepine with a short-term clinical effect $\left(T_{1 / 2}\right.$ in children is 2.5-4 h), which reduces anxiety and causes sedation and retrograde amnesia of the treatment. Midazolam has relatively higher volume of distribution $(V d)$ than other benzodiazepines due to its lipophilicity. In obese patients $T_{1 / 2}$ may increase from $2 \mathrm{~h}$ to $8 \mathrm{~h}$. The drug is metabolised in the liver by hydroxylation to its metabolite (1-hydroxymidazolam), which has $10 \%$ of the activity of the original drug. The authors are of the opinion that intravenous administration seems to be optimal, although oral, nasal or even anal administration may be equally effective, depending on the type of endoscopic procedure [20, 21]. The dosage of the drug strictly depends on the patient's age, body weight and way of administration. The titration regimen is necessary when the drug is intravenously administered to infants and small children, in order to avoid excessive and uncontrolled deepening of sedation [1, 21].

\section{Other benzodiazepines}

The half-life of diazepam is particularly long $\left(T_{1 / 2}\right.$ 0.8-2.25 days), especially in newborns, infants and obese patients (3.9-3.29 days). It is also decomposed into active metabolites with a long-term effect (nordiazepam, N-desmethyldiazepam). On the other hand, lorazepamis not useful in procedural sedation due to its late onset (15-20 min) and long-term effect (6-8 h).

\section{Other drugs}

In medical reference books it is possible to find more and more publications which say that non-anaesthesiologists use other anaesthetic drugs for sedation, e.g. ketamine, etomidate or propofol $[22,23]$. According to the Food and Drug Administration (FDA) guidelines, these drugs are registered as anaesthetics rather than sedatives or hypnotics. Apart from that, they have a narrow safety margin. The younger the patient is, the narrower the safety margin is. This requires that the doctor who administers these drugs intravenously should be very experienced, as the drugs are available only in intravenous form. An inexperienced doctor may inappropriately assess the course of sedation and this may lead to respiratory and circulatory disorders, which are a life hazard. Therefore, in 2010 all European anaesthesiological societies signed the Declaration of Helsinki, the primary goal of which was to guarantee appropriate conditions for patients to be safely an aesthetised [24]. According to the declaration, non-anaesthesiologist doctors applying those drugs, especially propofol, should be appropriately skilled in unblocking the airway and in resuscitation, and they are required to have certificates confirming their skills. Apart from that, some centres require certificates proving that the doctor has completed a theoretical and practical training course, which includes BLS training (Basic Life Support), ALS training (Advanced Life Support) and training in the operation of a defibrillator [25].

\section{Analgesics}

During painful endoscopic procedures of the alimentary tract it is necessary to apply opioid analgesic drugs, which have a short and strong effect, but they are burdened with some side effects and the risk of nausea, vomiting, aspiration and central respiratory depression. It is important to reduce the drug dosage by half in order to avoid the cumulative effect, especially when a due dose of a hypnotic drug was applied. In practice, the following drugs are most often applied: alfentanil, fentanyl or remifentanil, at the doses of $5 \mu \mathrm{g} / \mathrm{kg}$ of body weight, $1 \mu \mathrm{g} / \mathrm{kg}$ of body weight and $0.5-1.0 \mu \mathrm{g} / \mathrm{kg}$ of body weight, respectively. 


\section{Summary}

Although endoscopic procedures of the alimentary tract were successfully introduced into paediatric practice in the 1970s, in recent years their number has grown considerably, increasing the diagnostic and therapeutic capabilities in diseases of the alimentary tract in infants and children. These procedures should be carried out with sedation, so both the paediatrician and anaesthesiologist must have special skills, including recognition of anatomical, physiological and psychological differences. This guarantees higher safety of the procedure and reduces the risk of complications. The younger the child is, the greater the risk of complications is. Thus, close cooperation between the endoscopist and anaesthesiologist seems to be good practice, especially as far as children with congenital disorders and severe concomitant diseases are concerned.

\section{References}

1. Bartkowska-Śniatkowska A, Puklińska-Cieśla K, Ignyś I, et al. Endoscopic procedures in children - comfort and safety [Polish]. Prz Gastroenterol 2008; 3: 165-8.

2. American Society of Anesthesiologists. Taskforce on sedation and analgesia by non-anesthesiologists. Practiceguidelines for prevention, detection, and management of respiratory depression associated with neuraxial opioid administration. Anesthesiology 1996; 84: 459-70.

3. Larsen R. Anaesthesiology [Polish]. Urban \& Partner, Wroctaw 2008; 313-28.

4. Morton NS, Peutrel JM. Anaesthesiology and intensive therapy in children [Polish]. Urban \& Partner, Wrocław 2003; 52-87.

5. Schindler MB, Bohn D, Cox PN, et al. Outcome of out-of-hospital cardiac and respiratory arrest in children. N Engl J Med 1996; 335: 1473-9.

6. Becker DE, Haas DA. Management of complications during moderate and deep sedation. Respir Cardiovasc Consider 2007; 54: 59-69.

7. Pitteti RD, Singh S, Pierce MC. Safe and efficacious use of procedural sedation and analgesia by nonanesthesiologists in a pediatric emergency department. Arch Pediatr Adolesc Med 2003; 157: 1090-6.

8. Cote CJ, Karl HW, Notterman DA, et al. Adverse sedation events in pediatrics: a critical incident analysis of contributing factors. Pediatrics 2000; 106: 633-44.

9. Gorelick M, Nagler J, Losej JD, et al. Pediatric sedation pearls. Clin Ped Emerg Med 2007; 8: 268-78.

10. Cravero JP, Blike GT, Beach $M$, et al. Incidence and nature of adverse events during pediatric sedation/anesthesia for procedures outside the operating room: report from Pediatric Sedation Research Consortium. Pediatrics 2006; 118: 1087-96.

11. Cavill G, Kerr K. Preoperative management. In: Fundamentals of anesthesia. Smith T, Pinnock C, Lin T (eds.). Cambridge University Press 2009; 1-24.

12. Inal MT, Memis D, Kargi M, et al. Comparison of TrueView EVO2 with Miller laryngoscope in paediatric patients. Eur J Anaesthesiol 2010; 27: 950-4.
13. Burgoyne LL, Savege TM, Simpson BR, et al. How well do pediatric anesthesiologists agree assigning ASA physical status classifications to their patients? Paediatr Anaesth 2007; 17: 956-62.

14. Collins CE, Everett LL. Challenges in pediatric ambulatory anesthesia: kids are different. Anesthesiol Clin 2010; 28: 315-28.

15. Mallampati SR. Clinical sign to predict difficult tracheal intubation (hypothesis). Can Anaesth Soc J 1983; 30: 316-17.

16. Von Ungem-Sternberg BS, Boda K, Schwab C, et al. Laryngeal mask airway is associated with an increase incidence of adverse respiratory events in children with recent upper respiratory tract infections. Anesthesiology 2007; 107: 714-9.

17. Machotta A. Noncooperation and refusal during induction of anesthesia in children. Anesthesiol Intensivmed Noftallmed Achmerzther 2010; 6: 378-82.

18. Dere K, Sucullu I, Budak ET, et al, A comparison of dexmedetomidine versus midazolam for sedation, pain and hemodynamic control during colonoscopy under conscious sedation. Eur J Anaesthesiol 2010; 7: 648-52.

19. Vagnoli L, Caprili S, Messeri A. Parental presence, clowns or sedative premedication to treat preoperative anxiety in children: what could be the most promising option? Paediatr Anaesth 2010; 20: 937-43.

20. Wood M. The safety and efficacy of using a concentrated intranasal midazolam formulation for paediatric dental sedation. SAAD Dig 2011; 27: 16-23.

21. Robinson DN. Pediatric sedation technique. Curr Anesth Crit Care 2000; 11: 250-4.

22. Heuss LT, Scnieper P, Pfimlin E, et al. Nurse-administered sedation with propofol under observation of the endoscopist: prospective observation study with more than 500 patients. Gastrointest Endosc 2003; 57, poster 1481.

23. Patel KM, Simon HK, Stockwell CA, et al. Pediatric procedural sedation by a dedicated non anaesthesiology pediatric sedation service using propofol. Pediatr Emerg Care 2009; 25 : 133-8.

24. Mellin-Olsen J, Staender S, Whitaker DK, et al. The Helsinki Declaration on patient safety in anesthesiology. Eur J Anaesthesiol 2010; 27: 592-7.

25. Dumonceau JM, Riphaus A, Aparicio JR, et al. European Society of Gastrointestinal Endoscopy, European Society of Gatroenterology and Endoscopy Nurses and Associates, and European Society of Anaesthesiology Guidelines: non anaesthesiologist administration of propofol for Gl endoscopy. Eur J Anaesthesiol 2010; 27: 1016-30.

Received: 29.12 .2011

Accepted: 30.03 .2012 